\title{
Longer withdrawal time is not associated with increased patient discomfort in colonoscopy: a retrospective observational study
}

\author{
Kenichiro Majima, Yosuke Muraki \\ Department of Health Management, Kameda Medical Center, Kamogawa, Japan
}

Purpose: Withdrawal time of sufficient length is a quality indicator for colonoscopies. Nonetheless, whether extending the withdrawal time contributes to patient discomfort remains unknown. This study aimed to clarify the relationship between colonoscopy withdrawal time and patient discomfort.

Methods: A cohort of consecutive patients who underwent colonoscopy at a single institution from October 2018 to January 2020 was retrospectively analyzed. Initially, the relationship between the mean withdrawal time for each colonoscopist in no-finding examinations and polyp detection rate was investigated in 2,043 patients. Subsequently, the primary outcome of association between withdrawal time and patient discomfort, as determined by patient questionnaire, was assessed for each examination in 481 patients from the initial cohort.

Results: The mean withdrawal time was strongly correlated with polyp detection rate (correlation coefficient, 0.72 ; $\mathrm{P}<0.001)$. In contrast, longer withdrawal time was not associated with increased discomfort; however, there was a weak inverse correlation between patient discomfort and longer withdrawal time (correlation coefficient, $-0.25 ; \mathrm{P}<0.001$ ). Similarly, multiple regression analysis adjusted for confounding variables revealed that longer withdrawal time was not associated with increased patient discomfort (regression coefficient, -0.04 for each 1-minute increase in the length of withdrawal time; $\mathrm{P}=0.45$ ).

Conclusion: This study showed for the first time that longer withdrawal times did not result in increased discomfort, indicating that withdrawal time can be extended to sufficient length for optimal patient examination and polyp detection.

Keywords: Colonoscopy; Healthcare quality indicators; Pain; Polyps; Carbon dioxide

\section{INTRODUCTION}

High-quality colonoscopies are important for detecting polyps as well as ensuring that patients comply with subsequent examinations. The primary quality indicator of a colonoscopy is the adenoma detection rate (ADR), while the withdrawal time is another

Received: May 29, 2021 - Revised: Jun 29, 2021 - Accepted: Jul 12, 2021 Correspondence to: Kenichiro Majima, MD

Department of Health Management, Kameda Medical Center, 929 Higashicho, Kamogawa, Chiba 296-8602, Japan

Email: majima.kenichiro@kameda.jp

ORCID: https://orcid.org/0000-0001-9495-4873

(C) 2023 The Korean Society of Coloproctology

This is an open-access article distributed under the terms of the Creative Commons Attribution NonCommercial License (https://creativecommons.org/licenses/by-nc/4.0) which permits unrestricted noncommercial use, distribution, and reproduction in any medium, provided the original work is properly cited. important indicator $[1,2]$. An advantage of withdrawal time is that it can be determined immediately at the time of examination. Studies have suggested that performing the procedure with an adequate withdrawal time improves examination accuracy $[3,4]$, and colonoscopists with low ADRs are commonly advised to extend withdrawal time [1]. However, a longer withdrawal time might increase patient discomfort, which is of particular concern in the absence of sedation. Patient discomfort during endoscopic procedures not only reduces patient satisfaction but also reduces compliance in the next examination $[5,6]$. To the best of our knowledge, no study has investigated the relationship between longer withdrawal time and patient discomfort. This study aimed to clarify the relationship between colonoscopy withdrawal time and patient discomfort. 


\section{METHODS}

\section{Ethical statements}

The present study was conducted in accordance with the provisions of the Declaration of Helsinki (as revised in Fortaleza, Brazil, in October 2013) and was approved by the Institutional Review Board of our institution (No. 19-129-200225). Because this was a retrospective observational study using existing data and did not include invasive interventions, the requirement for informed consent from study patients was waived by the Institutional Review Board. However, written informed consent for colonoscopy was obtained. The study protocol was published on the hospital's website. This method was in accordance with the Japanese "Ethical Guidelines for Medical and Health Research Involving Human Subjects."

\section{Analysis of the association between withdrawal time and diagnostic accuracy Study population and measures}

In an initial analysis of withdrawal time and diagnostic accuracy, we investigated consecutive patients who underwent colonoscopy in opportunistic screening from October 2018 to January 2020. Patients were excluded if they were examined by a colonoscopist who had performed $<30$ cases during this period, had poor bowel preparation, and had undergone operation on the colon; the cecum was not reached; or the colonoscopist was changed during the examination (Fig. 1). Polyp detection rate (PDR) was used as an indicator of diagnostic accuracy. PDR is defined as the

Consecutive patients who underwent colonoscopy in opportunistic screening from October 2018 to January 2020 $(\mathrm{n}=2,273)$

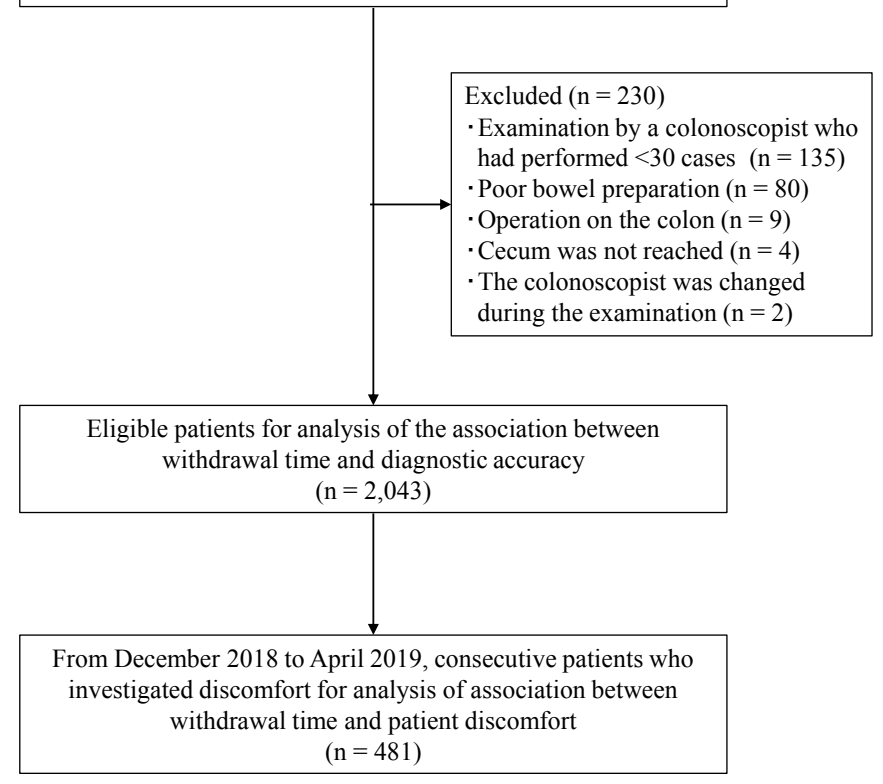

Fig. 1. Flow diagram of the study population. proportion of cases with a reported polyp out of all cases, with polyps including those of all types. Withdrawal time was determined as the time from when the scope reached the cecum to when it was removed from the anus, and the mean withdrawal time in normal colonoscopies (NWT) for each colonoscopist was used in the analysis.

\section{Colonoscopy procedure}

The scopes used were mainly PCF-H290I and PCF-Q260AI (Olympus), while a small-diameter long scope was used only rarely (PCF-PQ260L, Olympus). A short black hood was attached to the tip of the scope (MAJ-1990 or MAJ-1988, Olympus). Carbon dioxide $\left(\mathrm{CO}_{2}\right)$ was used for insufflation with a $\mathrm{CO}_{2}$ insufflator (Olympus UCR Insufflator). A sedative was administered upon the patient's request and with the physician's permission; when administered, an intravenous injection of pethidine was predominantly used. The decision to administer an antispasmodic agent depended on the colonoscopist; when administered, butylscopolamine or glucagon was injected intravenously. Any lesion detected was not resected at the time, and in one case requiring resection, this was performed on another day. In Japan, nonresection of diminutive polyps is permitted; thus, only polyps of $>5 \mathrm{~mm}$ were judged to require resection. Withdrawal time was measured with a stopwatch during the examination; the endoscopic technician declared the time, and the colonoscopist recorded it in the report. If withdrawal time was missing from the report, the time was determined by consulting the times on the cecum and anal images as they were written on the recorded images.

\section{Analysis}

We investigated the correlation coefficient between the mean NWT and PDR for each colonoscopist. Furthermore, a multivariate analysis was used to analyze the relationship between examinations performed by a colonoscopist with a mean NWT of $\geq 7$ minutes and increasing detection of one or more polyps. We adjusted for age, sex, presence or absence of previous unresected polyps, examinations performed by a colonoscopist with a median insertion time of $\leq 5$ minutes, and years of experience as a colonoscopist. The cutoff values for withdrawal time and insertion time in this multivariate analysis were the median values for each colonoscopist.

\section{Analysis of association between withdrawal time and patient discomfort Study population and measures}

The relationship between withdrawal time and patient discomfort was investigated in consecutive patients from December 2018 to April 2019, which is the 5 months within the above-mentioned target period. To investigate discomfort, a questionnaire was administered to patients at a different location from the endoscopy unit by staff other than the ones who performed the endoscopy. Discomfort was evaluated on a face scale of $0-10$ on a 6-level ques- 


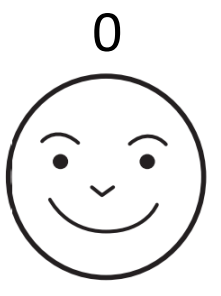

No discomfort
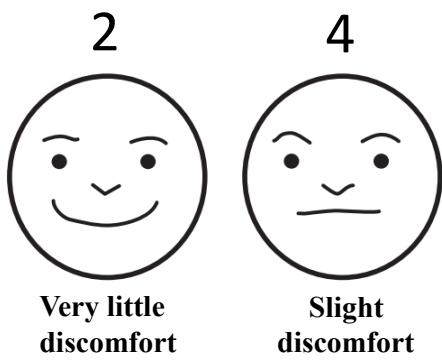
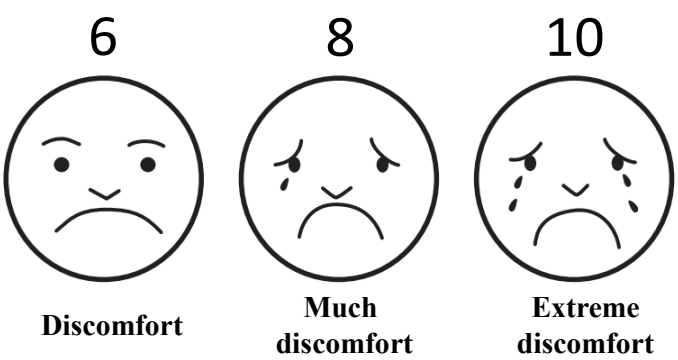

Fig. 2. Discomfort rating scale.

tionnaire (Fig. 2). Originally, the questionnaires were intended for improvement of hospital services to patients, and we used the results retrospectively. The withdrawal time used for this analysis was the withdrawal time for all examinations, including for cases with abnormal findings.

\section{Analysis}

We investigated the correlation coefficient between withdrawal time and patient discomfort for each examination. Furthermore, as the primary analysis, a multivariate analysis was used to analyze the relationship between withdrawal time and patient discomfort for each examination. We adjusted for confounding factors using the following explanatory factors in multiple regression analysis: sex [7-10], age $[8,9,11,12]$, body mass index $[8,9,11]$, history of colonoscopy $[8,9,11]$, history of uterine or ovarian surgery $[8,11]$, detected diverticula $[10]$, use of a long, thin scope [13], use of an antispasmodic agent $[8,12]$, use of a sedative [14], insertion time (each examination) $[7,8]$, and each individual colonoscopist [9].

As a sensitivity analysis to confirm the robustness of the results, some subgroup analyses were performed for cases examined by the physician with the largest number of cases, cases with a short insertion time ( $\leq 4.5$ minutes), and cases without sedation. Given the small number of cases, we only evaluated the correlation rather than perform multivariate analysis.

\section{Statistical analyses}

For the NWT for each colonoscopist, we used the mean value as described previously $[1,3]$. Otherwise, the mean was used for values with a normal distribution, and the median was used for values with a non-normal distribution. Spearman's rank correlation coefficient was used for correlation analysis. The correlation coefficient was defined as follows: $<0.2$ indicates no correlation, 0.2 to $<0.4$ indicates a weak correlation, 0.4 to $<0.7$ indicates a moderate correlation, and $\geq 0.7$ indicates a strong correlation. Logistic regression was used to determine the association between withdrawal time and polyp detection, and multiple regression was used to determine the association between withdrawal time and discomfort. The sample size was the largest number of cases available to maximize statistical power. All statistical analyses were per-
Table 1. Characteristics of 2,043 patients in the investigation of the correlation between withdrawal time and polyp detection rate

\begin{tabular}{lc}
\hline Characteristic & Data \\
\hline Age (yr) & $60 \pm 11^{\mathrm{a}}$ \\
Male sex & $1,341(65.6)^{\mathrm{b}}$ \\
No. of colonoscopists & 20 \\
Insertion time (min) & $4.5^{\mathrm{c}}$ \\
Withdrawal time in negative result & $(\mathrm{min})$ \\
Polyp detection rate (\%) & $6.9^{\mathrm{e}}$ \\
\hline
\end{tabular}

${ }^{\mathrm{a}}$ Mean \pm standard deviation. ${ }^{\mathrm{b}}$ Number (\%). ${ }^{\mathrm{c}}$ Median. ${ }^{\mathrm{d}} \mathrm{n}=502 .{ }^{\mathrm{e}}$ Mean.

formed using EZR ver. 1.52 (Saitama Medical Center, Jichi Medical University), which is a graphical user interface for $\mathrm{R}$ ( $\mathrm{R}$ Foundation for Statistical Computing, Vienna, Austria). A P-value of $<0.05$ was considered statistically significant.

\section{RESULTS}

\section{Withdrawal time and PDR}

A total of 2,043 eligible patients were included in the analysis of the association between withdrawal time and diagnostic accuracy (Fig. 1). Patient characteristics are shown in Table 1. Among the 20 colonoscopists who performed the examinations, the median years of experience was 12 years (range, 3-32 years), median PDR was $44.5 \%$ (range, $23.9 \%-73.6 \%$ ), and median NWT was 7.0 minutes (range, 4.5-10.1 minutes). The correlation between NWT and PDR as determined for individual colonoscopists was strong and significant (correlation coefficient, $0.72 ; \mathrm{P}<0.001$ ). In the logistic regression adjusted for various factors, the detection of polyps in examinations performed by a colonoscopist with a mean NWT of $\geq 7$ minutes was high (odds ratio, 4.51; $\mathrm{P}<0.001$ ) (Table 2).

\section{Withdrawal time and discomfort}

A total of 481 eligible patients were included in the multiple regression analysis to determine the association between withdrawal time and patient discomfort (Fig. 1). Patient characteristics are shown in Table 3. We found a weak inverse correlation be- 
Table 2. Logistic analysis results for factors related to polyp detection rate

\begin{tabular}{|c|c|c|}
\hline Variable & $\mathrm{OR}(95 \% \mathrm{Cl})$ & P-value \\
\hline Male sex & $1.76(1.43-2.17)$ & $<0.001$ \\
\hline Age & $1.02(1.01-1.03)$ & $<0.001$ \\
\hline Unresected polyp in a previous colonoscopy & $5.11(4.04-6.47)$ & $<0.001$ \\
\hline $\begin{array}{l}\text { Examination performed by a colonoscopist } \\
\text { whose mean withdrawal time is } \geq 7 \mathrm{~min} \text { in } \\
\text { normal colonoscopies }\end{array}$ & $4.51(3.59-5.65)$ & $<0.001$ \\
\hline $\begin{array}{l}\text { Examination performed by a colonoscopist } \\
\text { whose median insertion time is } \leq 5 \mathrm{~min}\end{array}$ & $2.37(1.86-3.03)$ & $<0.001$ \\
\hline Colonoscopist's experience in colonoscopy (yr) & $1.02(1.00-1.03)$ & 0.016 \\
\hline
\end{tabular}

$\mathrm{OR}$, odds ratio; $\mathrm{Cl}$, confidence interval.

Table 3. Characteristics of 481 patients in the investigation of the correlation between withdrawal time and patient discomfort

\begin{tabular}{|c|c|}
\hline Characteristic & Data \\
\hline Age (yr) & $60 \pm 12$ \\
\hline Male sex & $314(65.3)$ \\
\hline Body mass index $\left(\mathrm{kg} / \mathrm{m}^{2}\right)$ & $23.9(21.6-26.0)$ \\
\hline Patients with colonoscopic experience & 381 (79.2) \\
\hline History of uterine or ovarian surgery & $44(9.2)$ \\
\hline Detected diverticulum & $148(30.1)$ \\
\hline No. of colonoscopists & 20 \\
\hline Use of small-diameter long scope & $22(4.6)$ \\
\hline Antispasmodic agent use & $409(85.0)$ \\
\hline Sedative use & $219(45.5)$ \\
\hline Meperidine alone ${ }^{\mathrm{a}}$ & $197(41.0)$ \\
\hline Midazolam alone ${ }^{b}$ & $10(2.1)$ \\
\hline Meperidine and midazolam ${ }^{c}$ & $12(2.5)$ \\
\hline Insertion time (min) & $4.5(3-6)$ \\
\hline Withdrawal time (min) & $7.5(6-10)$ \\
\hline Discomfort score ${ }^{\mathrm{d}}$ & $2(0-4)$ \\
\hline
\end{tabular}

Values are presented as mean \pm standard deviation, number (\%), number only, or median (interquartile range).

${ }^{a-c}$ Median dosage (mg): ${ }^{a} 35,{ }^{b} 2,{ }^{c}$ meperidine $35 \mathrm{mg}$, midazolam $1.25 \mathrm{mg}$. ${ }^{\mathrm{d}}$ Range, 0-10.

tween withdrawal time and patient discomfort, as determined for each examination, which was significant (correlation coefficient, -0.25 ; $\mathrm{P}<0.001$ ) (Fig. 3). In the multiple regression analysis adjusted for various confounding factors, no significant correlation was found between withdrawal time and patient discomfort as determined for each examination (regression coefficient, -0.04 ; $\mathrm{P}=0.45$ ) (Table 4).

In a sensitivity analysis performed on subgroups of cases, we found no positive correlation between withdrawal time and patient discomfort for cases examined by the physician with the

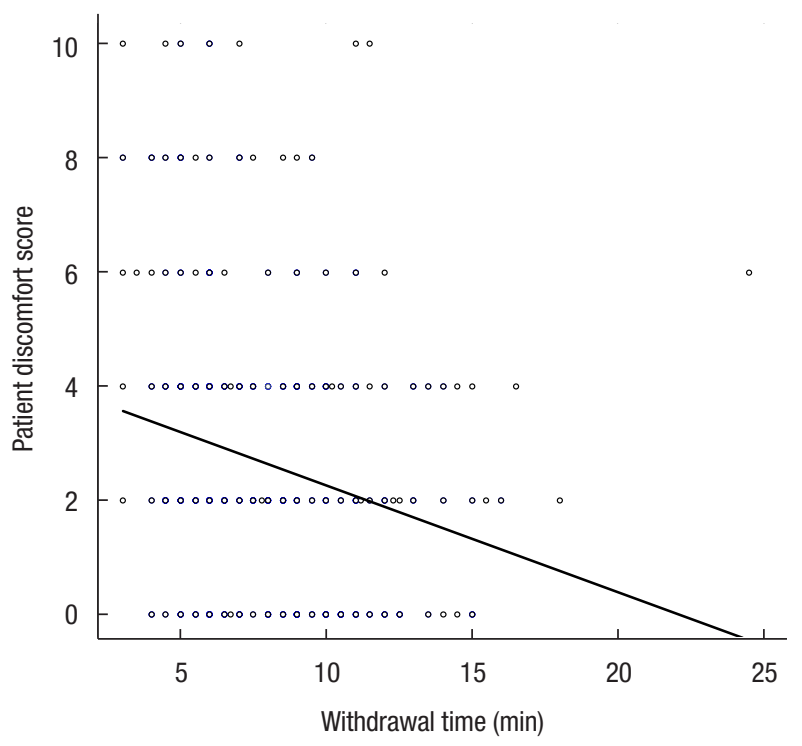

Fig. 3. Correlation between withdrawal time and patient discomfort for each examination.

largest number of cases $(n=107)$, cases with an insertion time of $\leq 4.5$ minutes $(n=257)$, and cases without sedation $(n=262)$. The correlation coefficients were $-0.06(\mathrm{P}=0.512),-0.23(\mathrm{P}<0.001)$, and $-0.22(\mathrm{P}<0.001)$ for these groups, respectively, with a significant weak inverse correlation in cases with an insertion time of $\leq 4.5$ minutes and in cases without sedation.

\section{DISCUSSION}

In this study, withdrawal time showed a strong positive correlation with PDR. Moreover, longer withdrawal time did not increase patient discomfort. Previous studies have shown that longer withdrawal time correlates with increased ADR and decreased the rate of interval cancer $[3,4]$; however, there have also been reports in which longer withdrawal time did not increase ADR [15] and did not correlate with PDR [16]. If the withdrawal time is not correlated with the lesion detection rate, the withdrawal time cannot be used as a quality indicator. Therefore, the present study first confirmed that the withdrawal time and PDR were positively correlated and that the withdrawal time served as a quality indicator at our institution. In previous reports, the reason for the inconsistent relationship between withdrawal time and lesion detection was not elucidated; however, it is assumed that increased lesion detection rate requires longer withdrawal time with careful inspection, such as inspection of the back of colonic folds, and that longer withdrawal time without careful inspection does not result in increased lesion detection rate $[17,18]$. Since our study showed a strong correlation between withdrawal time and PDR, we can theorize that colonoscopists with longer withdrawal times performed examinations more carefully.

Although there was concern that longer withdrawal time would 
Table 4. Impact of factors on patient discomfort by multiple regression analysis

\begin{tabular}{|c|c|c|}
\hline Factor & $\begin{array}{l}\text { Regression coefficient } \\
\qquad(95 \% \mathrm{Cl})\end{array}$ & P-value \\
\hline Age $(1+)$ & $-0.03(-0.05$ to -0.01$)$ & $<0.001$ \\
\hline Male sex & $-0.75(-1.25$ to -0.25$)$ & 0.003 \\
\hline Body mass index $(1+)$ & $-0.03(-0.09$ to 0.02$)$ & 0.27 \\
\hline Patients without colonoscopic experience & $0.31(-0.21$ to 0.82$)$ & 0.25 \\
\hline History of uterine or ovarian surgery & 0.66 (-0.1 to 1.42) & 0.089 \\
\hline Detected diverticulum & $0.41(-0.03$ to 0.85$)$ & 0.066 \\
\hline Use of small-diameter long scope & $-0.28(-1.27$ to 0.71$)$ & 0.58 \\
\hline Antispasmodic agent use & -0.07 (-0.78 to 0.65$)$ & 0.85 \\
\hline Sedative use & $-0.98(-1.4$ to -0.56$)$ & $<0.001$ \\
\hline Insertion time (1 min +) & $0.17(0.11$ to 0.23$)$ & $<0.001$ \\
\hline Withdrawal time $(1 \mathrm{~min}+)$ & $-0.04(-0.14$ to 0.06$)$ & 0.45 \\
\hline \multicolumn{3}{|l|}{ MD No. } \\
\hline 1 & $-0.36(-1.7$ to 0.97$)$ & 0.59 \\
\hline 2 & $-0.56(-2.43$ to 1.31$)$ & 0.56 \\
\hline 3 & $-1.01(-2.02$ to 0.002$)$ & 0.05 \\
\hline 4 & $-1.09(-2.49$ to 0.32$)$ & 0.13 \\
\hline 5 & $-1.12(-2.23$ to 0.003$)$ & 0.049 \\
\hline 6 & $-1.39(-2.76$ to -0.02$)$ & 0.047 \\
\hline 7 & $-1.40(-2.37$ to -0.43$)$ & 0.005 \\
\hline 8 & $-1.60(-3.02$ to -0.18$)$ & 0.027 \\
\hline 9 & $-1.74(-3.34$ to -0.14$)$ & 0.033 \\
\hline 10 & $-1.88(-2.95$ to -0.82$)$ & $<0.001$ \\
\hline 11 & $-2.25(-3.21$ to -1.3$)$ & $<0.001$ \\
\hline 12 & $-2.25(-3.78$ to -0.73$)$ & 0.004 \\
\hline 13 & $-2.32(-3.81$ to -0.83$)$ & 0.002 \\
\hline 14 & $-2.33(-3.38$ to -1.28$)$ & $<0.001$ \\
\hline 15 & $-2.37(-3.45$ to -1.29$)$ & $<0.001$ \\
\hline 16 & $-2.74(-4.35$ to -1.12$)$ & $<0.001$ \\
\hline 17 & $-2.93(-4.47$ to -1.38$)$ & $<0.001$ \\
\hline 18 & $-2.94(-4.16$ to -1.72$)$ & $<0.001$ \\
\hline 19 & $-3.12(-4.83$ to -1.41$)$ & $<0.001$ \\
\hline
\end{tabular}

$\mathrm{Cl}$, confidence interval; $\mathrm{MD}$, medical doctor.

increase patient discomfort, surprisingly, we did not find a correlation between these in the present study. A previous study, although one that did not explicitly investigate the relationship between withdrawal time and discomfort, found that discomfort was high in the first half and low in the second half of the examination [10], supporting our findings. In addition, presence or absence of polyps was reportedly not associated with discomfort in colonoscopy without resection of detected lesions, as performed in the present study [12]. Because cases with polyps should have longer withdrawal time, this study also supports our findings. We found a lack of correlation between withdrawal time and patient discomfort in both our multivariate analyses adjusted for various factors and subgroup analyses, demonstrating the robustness of our findings and the likelihood that they will be generalizable to other populations. The lack of correlation described herein is important given the relationship between patient comfort and compliance with endoscopic procedures and the need for sufficient time to perform a thorough examination. Lesion detection rate has been reported to increase up to a withdrawal time of approximately 10 minutes [19], and the UK National Health Service's guideline stipulates a target withdrawal time of $\geq 10$ minutes [20]. Colonoscopists should take sufficient time to inspect carefully, which, on the basis of the present study, can be done without increasing discomfort for withdrawal times of up to approximately 10 minutes.

One potential confounding factor we considered was an inverse correlation between withdrawal time and insertion time [21]. If these times are correlated, shorter insertion times could result in less discomfort and longer withdrawal times. However, in the present study, no inverse correlation was found between insertion time and withdrawal time (data not shown). In addition, multivariate analysis adjusted for factors including insertion time and subgroup analysis for groups with short insertion times showed that longer withdrawal time was not associated with increased discomfort. Therefore, it is unlikely that our results reflect confounding from such a relationship.

It is not clear why increasing withdrawal time did not result in an increase in discomfort; however, we speculate that the use of $\mathrm{CO}_{2}$ insufflation is one important reason, as this has previously been shown to reduce discomfort [22].

Several factors have been reported to be associated with discomfort in colonoscopy: while many studies have consistently shown high discomfort in women [7-10], the association with other factors varies [7-12]. In the present study, factors that were significantly associated with increased patient discomfort were female sex, young age, nonuse of sedative, and examination by some individual colonoscopists. In addition, longer insertion time was a significant factor in high levels of discomfort. The association of these factors with discomfort has also been reported in previous studies [7-10, 12,14]. Although some colonoscopists may wish to avoid longer withdrawal times because of increased discomfort, our results indicate that withdrawal time is not the main cause of discomfort, suggesting that such adjustments are unnecessary and that withdrawal time can be extended such that sufficient careful inspection can be performed. Instead, smoother insertion is key to reducing discomfort.

This study has some limitations. First, this is a retrospective observational study in which unpredictable confounding effects may have existed. Therefore, we adjusted for various factors to minimize these confounding effects. Second, this is a single-center study, and the results may therefore not be as generalizable as 
those from a multicenter study. Similar analysis in additional cases and other cohorts will be necessary to further characterize the relationship.

In conclusion, we report that longer withdrawal times in colonoscopy do not contribute to patient discomfort. This represents an important finding as it should allow for optimization of the colonoscopy procedure to focus on polyp detection.

\section{CONFLICT OF INTEREST}

No potential conflict of interest relevant to this article was reported.

\section{FUNDING}

None.

\section{REFERENCES}

1. Valori R, Rey JF, Atkin WS, Bretthauer M, Senore C, Hoff G, et al. European guidelines for quality assurance in colorectal cancer screening and diagnosis. First edition: quality assurance in endoscopy in colorectal cancer screening and diagnosis. Endoscopy 2012;44 Suppl 3:SE88-105.

2. Rex DK, Schoenfeld PS, Cohen J, Pike IM, Adler DG, Fennerty $\mathrm{MB}$, et al. Quality indicators for colonoscopy. Gastrointest Endosc 2015;81:31-53.

3. Barclay RL, Vicari JJ, Doughty AS, Johanson JF, Greenlaw RL. Colonoscopic withdrawal times and adenoma detection during screening colonoscopy. N Engl J Med 2006;355:2533-41.

4. Shaukat A, Rector TS, Church TR, Lederle FA, Kim AS, Rank JM, et al. Longer withdrawal time is associated with a reduced incidence of interval cancer after screening colonoscopy. Gastroenterology 2015;149:952-7.

5. Ko HH, Zhang H, Telford JJ, Enns R. Factors influencing patient satisfaction when undergoing endoscopic procedures. Gastrointest Endosc 2009;69:883-91.

6. Viiala $\mathrm{CH}$, Olynyk JK. Reasons for noncompliance with fiveyearly screening flexible sigmoidoscopy. Patient Prefer Adherence 2008;2:27-33.

7. Kim WH, Cho YJ, Park JY, Min PK, Kang JK, Park IS. Factors affecting insertion time and patient discomfort during colonoscopy. Gastrointest Endosc 2000;52:600-5.

8. Takahashi Y, Tanaka H, Kinjo M, Sakumoto K. Prospective evaluation of factors predicting difficulty and pain during sedationfree colonoscopy. Dis Colon Rectum 2005;48:1295-300.

9. Bugajski M, Wieszczy P, Hoff G, Rupinski M, Regula J, Kaminski MF. Modifiable factors associated with patient-reported pain during and after screening colonoscopy. Gut 2018;67:1958-64.

10. Elphick DA, Donnelly MT, Smith KS, Riley SA. Factors associated with abdominal discomfort during colonoscopy: a prospective analysis. Eur J Gastroenterol Hepatol 2009;21:1076-82.

11. Chung YW, Han DS, Yoo KS, Park CK. Patient factors predictive of pain and difficulty during sedation-free colonoscopy: a prospective study in Korea. Dig Liver Dis 2007;39:872-6.

12. Suzuki H, Nakamura M, Yamamura T, Maeda K, Sawada T, Mizutani Y, et al. A prospective study of factors associated with abdominal pain in patients during unsedated colonoscopy using a magnifying endoscope. Intern Med 2020;59:1795-801.

13. Sato K, Ito S, Shigiyama F, Kitagawa T, Hirahata K, Tominaga K, et al. A prospective randomized study on the benefits of a new small-caliber colonoscope. Endoscopy 2012;44:746-53.

14. ASGE Standards of Practice Committee; Early DS, Lightdale JR, Vargo JJ 2nd, Acosta RD, Chandrasekhara V, et al. Guidelines for sedation and anesthesia in GI endoscopy. Gastrointest Endosc 2018;87:327-37.

15. Sawhney MS, Cury MS, Neeman N, Ngo LH, Lewis JM, Chuttani $\mathrm{R}$, et al. Effect of institution-wide policy of colonoscopy withdrawal time $>$ or $=7$ minutes on polyp detection. Gastroenterology 2008;135:1892-8.

16. Moritz V, Bretthauer M, Ruud HK, Glomsaker T, de Lange T, Sandvei $\mathrm{P}$, et al. Withdrawal time as a quality indicator for colonoscopy: a nationwide analysis. Endoscopy 2012;44:476-81.

17. Barclay RL, Vicari JJ, Greenlaw RL. Effect of a time-dependent colonoscopic withdrawal protocol on adenoma detection during screening colonoscopy. Clin Gastroenterol Hepatol 2008;6:10918.

18. Lee RH, Tang RS, Muthusamy VR, Ho SB, Shah NK, Wetzel L, et al. Quality of colonoscopy withdrawal technique and variability in adenoma detection rates (with videos). Gastrointest Endosc 2011;74:128-34.

19. Lee TJ, Blanks RG, Rees CJ, Wright KC, Nickerson C, Moss SM, et al. Longer mean colonoscopy withdrawal time is associated with increased adenoma detection: evidence from the Bowel Cancer Screening Programme in England. Endoscopy 2013;45:20-6.

20. Public Health England. Bowel cancer screening programme standards: valid for data collected from 1 April 2018 [Internet]. Public Health England; c2021 [cited 2021 Apr 5]. Available from: https:// www.gov.uk/government/publications/bowel-cancer-screeningprogramme-standards/bowel-cancer-screening-programmestandards-valid-for-data-collected-from-1-april-2018\#bcsps14-interventiontreatment-scope-withdrawal-time

21. Benson ME, Reichelderfer M, Said A, Gaumnitz EA, Pfau PR. Variation in colonoscopic technique and adenoma detection rates at an academic gastroenterology unit. Dig Dis Sci 2010;55:16671.

22. Rogers AC, Van De Hoef D, Sahebally SM, Winter DC. A metaanalysis of carbon dioxide versus room air insufflation on patient comfort and key performance indicators at colonoscopy. Int J Colorectal Dis 2020;35:455-64. 